Copyright (C1996, American Institute of Aeronautics and Astronautics, Inc.

AIAA Meeting Papers on Disc, July 1996

A9637200, AIAA Paper 96-3066

Fission-assisted or self-sustaining gasdynamic mirror propulsion system

\author{
T. Kammash \\ Michigan Univ., Ann Arbor \\ M.-J. Lee \\ Michigan Univ., Ann Arbor
}

\begin{abstract}
AIAA, ASME, SAE, and ASEE, Joint Propulsion Conference and Exhibit, 32nd, Lake Buena Vista, FL, July 1-3, 1996
\end{abstract}

\begin{abstract}
A controlled fusion system that can be achieved with almost present-day or near-term technology is proposed as a propulsion device that could be utilized for interplanetary and interstellar space exploration. It is based on the Gasdynamic Mirror (GDM) which, when operated as a propulsion engine, is capable of producing specific impulses in excess of 200,000 seconds, and thrusts of tens of kilonewtons. This capacity stems from the fact that, at a sufficiently high plasma density, the collision mean free path is significantly shorter than the machine length, allowing the plasma to behave much like a fluid with confinement properties that are substantially different from those which characterize the standard collisionless mirror. Under these circumstances the plasma escape from the end is analogous to the flow of a fluid into a vacuum from a vessel with a hole. Using an appropriate set of conservation equations we have deduced the plasma parameters that underlie the propulsive capability of GDM. When applied to a manned mission to Pluto, it is shown that with GDM a round trip to the outermost planet in the solar system can be done in slightly over four years. (Author)
\end{abstract}




\title{
FISSION-ASSISTED OR SELF-SUSTAINING GASDYNAMIC MIRROR PROPULSION SYSTEM
}

\author{
T. Kammash* and M.-J. Lee† \\ Department of Nuclear Engineering and Radiological Sciences \\ The University of Michigan \\ Ann Arbor, MI 48109
}

\begin{abstract}
A controlled fusion system that can be achieved with almost present-day or near-term technology is proposed as a propulsion device that could be utilized for interplanetary and interstellar space exploration. It is based on the Gasdynamic Mirror (GDM) which, when operated as a propulsion engine, is capable of producing specific impulses in excess of 200,000 seconds, and thrusts of tens of kilonewtons. This capacity stems from the fact that at a sufficiently high plasma density the collision mean free path is significantly shorter than the machine length, allowing the plasma to behave much like a fluid with confinement properties that are substantially different from those which characterize the standard collisionless mirror. Under these circumstances the plasma escape from the end is analogous to the flow of a fluid into a vacuum from a vessel with a hole. Using an appropriate set of conservation equations we have deduced the plasma parameters that underlie the propulsive capability of GDM. We have also carried out a preliminary engineering analysis to estimate the masses of the major components, and have concluded that such a system can be constructed with present day, or near term technology that has been developed by the world effort in terrestrial fusion power research. When applied to a manned mission to Pluto, it is shown that with GDM a round trip to the outermost planet in the solar system can be done in slightly over four years.
\end{abstract}

\section{Introduction}

Missions, particularly manned ones, to the outer solar system and beyond require propulsion systems that would allow such undertakings to take place-in relatively short times-in order- to minimize exposure to galactic radiation, and to preserve the physical and mental health of the explorers who man them. Chemical propulsion is totally inadequate for such missions, and next to antimatter annihilation propulsion which is deemed to be not-too-near term, fusion-driven systems perhaps provide the most immediate approaches that can readily meet these objectives. Several such systems have been proposed in recent years $[1,2]$ but most of them can be disregarded because they may be viewed as requiring technologies that will not be ready in the foreseeable future. The exception is the Gasdynamic Mirror (GDM) [3] which can be considered as a present-day or near-term achievable system because it makes use of physics research and engineering developments that have been carried out for the past several decades in conjunction with worldwide effort to develop terrestrial fusion power. It utilizes a high density plasma that makes the collision mean free path of its charged particle constituents much shorter than its length, thereby making the plasma behave much like a fluid with confinement properties that are significantly different than those that characterize terrestrial power counterparts. Under these circumstances the escape of the plasma from the system is analogous to the flow of a gas into a vacuum from a vessel with a hole. The GDM propulsion engine is basically a simple magnetic mirror fusion reactor in which a fusion fuel (e.g., deuterium and tritium, DT) is injected at an appropriate energy, and confined for a sufficiently long time to allow fusion nuclear reactions to take place. Confinement is provided by the magnetic field which is made stronger at the ends of the cylindrical chamber than at the center, thus providing the desired reflecting force that prevents most of the particles from escaping through the end. In spite of that a significant fraction of the

*Professor and AIAA Associate Fellow

†Research Fellow in Nuclear Engineering 
fusion ions do escape carrying with them sufficient amounts of energy to render the simple mirror unacceptable as a potential terrestrial power reactor. Ironically, however, this leakiness is the very quality that makes it especially attractive as a propulsion system. By making GDM a high aspect ratio (length much larger than diameter) device, one of the magnetic mirrors can serve as a magnetic nozzle which will eject the plasma particles at very high speeds that generate very impressive propulsive capabilities. The other mirror could be utilized to transform the energy of the escaping particles directly into electric power. When the power coming from this direct converter is combined with that resulting from the thermal conversion of the power of the neutrons produced by the fusion reactions, the result can be sufficiently large electric power to make the system self-sustaining and obviating the need for an external power source. In the remaining portion of this paper we will present a brief discussion of the underlying physics, and some engineering considerations of GDM as a propulsion device, and then assess its propulsive capability by applying the results to missions to Mars, Jupiter and Pluto.

\section{Analysis and Technical Considerations}

We noted above that GDM is a simple magnetic mirror which when operated as a propulsion device will contain a sufficiently dense plasma for which the collision mean free path, $\lambda$, will be significantly shorter than the length, $L$, as illustrated in Fig. 1. The plasma flux across the mirror cross section, $A_{0}$, can be estimated as $A_{0} n v_{\text {th }}$ where $n$ is the plasma density and $v_{t h}$ is the particle thermal velocity. By dividing the total number of particles in the system, i.e., $A_{c} n L\left(A_{\mathrm{c}}\right.$ is the cross sectional area of the plasma in the central region) by the flux we can calculate the particle lifetime or the plasma confinement time, i.e.,

$$
\tau=\frac{A L}{A_{0} v_{t h}}=\frac{R L}{v_{\text {th }}}
$$

where $R$ is the magnetic mirror ratio seen by the plasma which in turn is related to the vacuum mirror ratio $R_{0}$ by

$$
R=\frac{R_{0}}{\sqrt{1-\beta}}
$$

The quantity $\beta$ given by

$$
\beta=\frac{\sum n T}{B_{p 0}{ }^{2} / 8 \pi} ; \quad B_{p}=B_{p 0} \sqrt{1-\beta}
$$

is the ratio of the plasma pressure to the (vacuum) magnetic field pressure and its value is a measure of how effective the magnetic field is in confining the plasma. A large value $(\beta \leq 1)$ usually indicates a stable plasma confinement and, because of its proportionality to the density $n$, it also is a measure of how large a power density the system is capable of sustaining.

In contrast with the classical terrestrial mirror machine where $\lambda>L$, Eq. (1) shows that the confinement time is a linear, rather than a logarithmic function of $R$. Consequently, in the gasdynamic mirror, examined here as a propulsion device, increasing the mirror ratio to the highest value permitted by the technology provides a much greater effect than in the classical mirror. For reasons that will become clear shortly, we consider systems for which $R \gg 1$, and when this is taken into account the condition for the validity of Eq. (1) becomes $\lambda / R \ll L$ which is much less stringent than $\lambda \ll<L$ cited earlier. In other words the quantity to be compared with the length of the device, $L$, is not the mean free path, but an effective mean free path against scattering through an angle of the order of the loss cone angle, $\theta$ $1 / \sqrt{R}$. Under these conditions any loss cone instability [4] arising from depletion of the velocity distribution will not have a serious adverse effect on the longitudinal confinement of the plasma-a salutary effect for the gasdynamic mirror propulsion concept. Any reduction in the scattering length cannot cause a significant change in the rate at which the plasma escapes through the mirrors; thus it cannot alter in a measurable way the confinement time given by Eq. (1). Another important feature of GDM is the high plasma density in the region just beyond the mirror (the expansion region of the nozzle) where it is comparable to that at the center of the system. Thus feature makes it possible to suppress the flute instability [5] which has plagued the classical axisymmetric mirror. Stability against this 

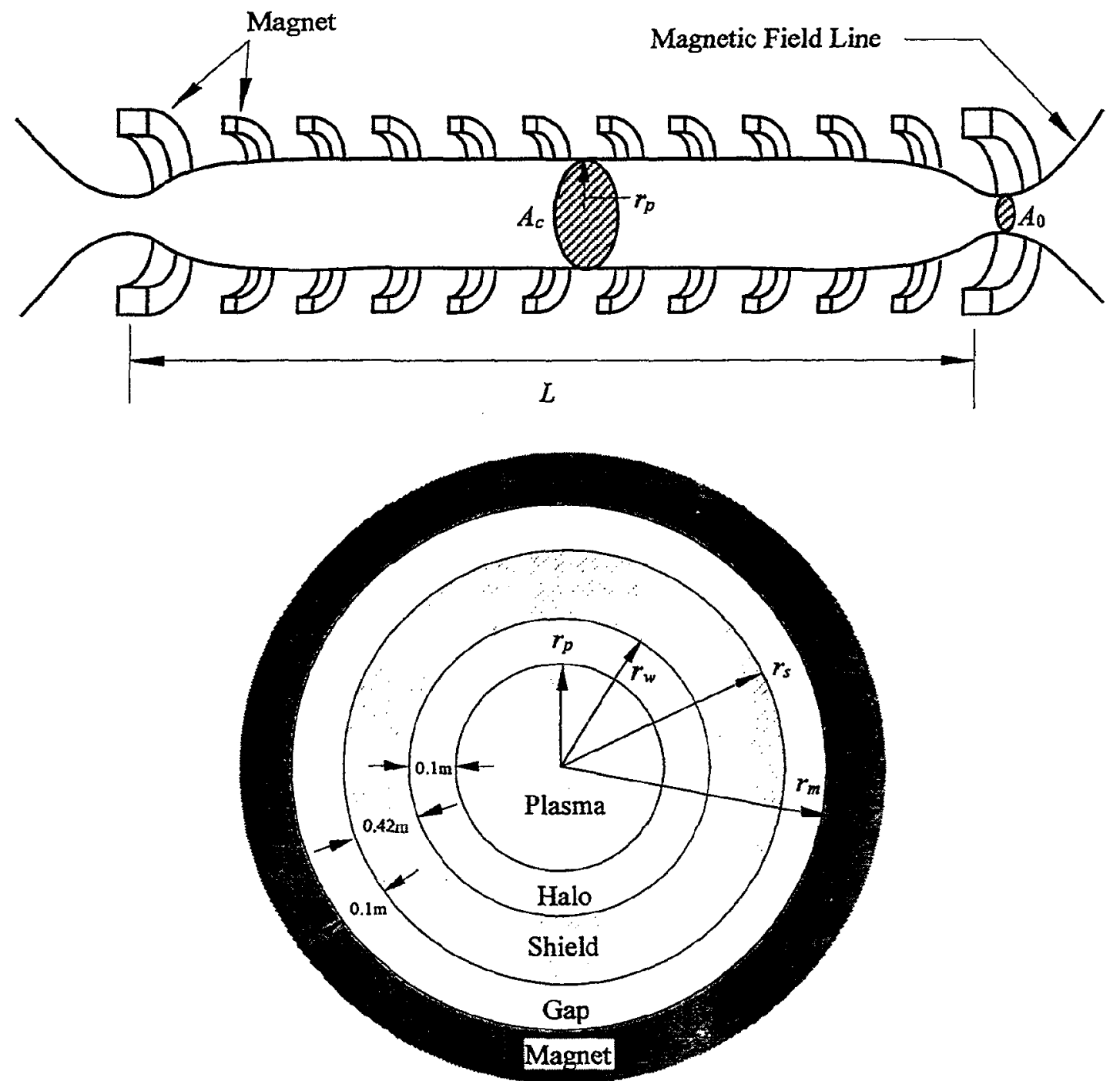

Fig. 1. Schematic and cross-sectional view of the gasdynamic fusion propulsion system

magnetohydrodynamic (MHD) mode and against the loss cone instability alluded to above, provide an added dimension to the utility of the gasdynamic mirror as an effective propulsion concept.

In order to assess the propulsive performance of_GDM_we will employ a simplified model [3] that consists of the following two steady state equations for the conservation of mass and energy in the system:

$$
\begin{gathered}
S-\frac{n}{\tau}-\frac{n^{2}}{2}\langle\sigma\rangle=0 \\
S E_{\text {in }}+\frac{n^{2}}{4}\left\langle\sigma \nu E_{c}-\frac{n E_{L}}{\tau}-P_{b}-P_{s}=0\right.
\end{gathered}
$$

In these equations $S$ represents the rate of injection of fuel ions per unit volume, $\langle\sigma\rangle\rangle$ the velocity- 
averaged fusion reaction cross section, $E_{\text {in }}$ the energy of the injected particles, $E_{c}$ the portion of energy produced by the fusion reaction that remains in the plasma (3.5 $\mathrm{MeV}$ in the case of DT), $P_{b}$ the bremsstrahlung radiation power, $P_{s}$ the synchrotron radiation power, and $E_{L}$ the mean energy of an escaping ion which for a maxwellian distribution has a value of $2 T$ with $T$ being the temperature. When the appropriate expressions for $P_{b}$ and $P_{s}[6]$ are injected into Eq. (5), and use is made of the definition of the gain factor $Q$, namely,

$$
Q=\frac{n^{2}}{4}\left\langle\sigma v E_{0} / \frac{n E_{i n}}{\tau}=\frac{P_{f}}{P_{i n j}}\right.
$$

with $E_{0}$ being the energy produced by fusion (e.g., $17.6 \mathrm{MeV}$ for DT), $P_{f}$ fusion power, and $P_{i n j}$, injection power, the balance equations can be solved for the length $L$ to yield

$$
L=\frac{\sqrt{8}\left(E_{\text {in }}-E_{L}\right)}{n R \sqrt{\pi m}\left[p_{0}+s_{0} T^{2 / 3}-\frac{1}{4}\langle\sigma\rangle\left(2 E_{\text {in }}+E_{0}\right) T^{-/ / 2}\right]}
$$

where $p_{0}$ and $s_{0}$ are constants and $m$ the ion mass. The above equation reveals some interesting facts concerning GDM, the most significant of which is that the length decreases at higher plasma densities and at larger mirror ratios, and the use of heavier ions, i.e., larger $m$, also results in shorter systems although this effect is quite negligible for most fusion fuels of interest. Since the validity of these results depends critically on the performance of the machine as a gasdynamic system, i.e., one for which $\lambda / R \ll L$, with $\lambda$ given by

$$
\lambda=\frac{3}{4} \sqrt{\frac{3}{2 \pi}} \frac{T^{2}}{n e^{4} \ln \Lambda}=1.253 \times 10^{18} \frac{T^{2}(\mathrm{keV})}{n\left(\mathrm{~cm}^{-3}\right)}(\mathrm{cm})
$$

it can be shown that $n \geq 10^{15} \mathrm{~cm}^{-3}, T \cong 10 \mathrm{keV}$ and $R \geq 50$ easily satisfy the above condition, and simultaneously yield acceptable lengths. A preliminary engineering design [3] using the configuration shown in Fig. 1 and utilizing magnet designs that range from present-day technology of $50 \mathrm{MA} / \mathrm{m}^{2}$ to superconducting magnets ( 250 $\mathrm{MA} / \mathrm{m}^{2}$ ), including radiators capable of ejecting heat at the rate of $5 \mathrm{MW} / \mathrm{Mg}$, is carried out to estimate the total mass of the vehicle. An example

Table 1

Performance of the gasdynamic mirror fusion propulsion system

\begin{tabular}{|l|r|l|}
\hline Parameter & \multicolumn{1}{|c|}{ Value } & Unit \\
\hline Gain factor & 1.22 & \\
Plasma density & $1 \times 10^{16}$ & $\mathrm{~cm}^{-3}$ \\
Plasma temperature & 10 & $\mathrm{keV}$ \\
Plasma mirror ratio & 100 & \\
Magnetic field (vacuum) & 9.207 & $\mathrm{~T}$ \\
Plasma length & 43.7 & $\mathrm{~m}$ \\
Plasma radius & 5 & $\mathrm{~cm}$ \\
Confinement time & $4.06 \times 10^{-3}$ & $\mathrm{sec}$ \\
Thrust & $2.52 \times 10^{3}$ & $\mathrm{~N}$ \\
Thrust power & $1.35 \times 10^{3}$ & $\mathrm{MW}$ \\
Injection power & $2.24 \times 10^{3}$ & $\mathrm{MW}$ \\
Fusion power & $2.73 \times 10^{3}$ & $\mathrm{MW}$ \\
Engine mass & 101 & $\mathrm{Mg}$ \\
Thermal converter mass & 44 & $\mathrm{Mg}$ \\
Direct converter mass & 27 & $\mathrm{Mg}$ \\
Radiator mass & 241 & $\mathrm{Mg}$ \\
Total vehicle mass & 413 & $\mathrm{Mg}$ \\
Specific power & 13 & $\mathrm{~kW} / \mathrm{kg}$ \\
Specific impulse & $1.27 \times 10^{5}$ & $\mathrm{sec}$ \\
\hline
\end{tabular}


of such analysis is given in Table 1 where we note that the total mass of the vehicle can be as low as $413 \mathrm{mT}$ with an engine mass and thrust power that yield a specific power of about $13-$ a value considered to be very attractive for fusion propulsion.

One glaring result for the system in Table 1 , however, is the large injection power required to operate this fusion rocket. Such power can perhaps be provided only by another nuclear system such as a fission reactor which if used would truly exacerbate the total mass of the system and perhaps eliminate it from consideration. To address this issue we have examined the conditions under which the system can be self-sustaining and not requiring an external power source to provide the power needed for continuous operation. For such analysis we refer to the power flow diagram illustrated in Fig. 2. As shown, the propulsion engine consists of the fusion reactor and the injector which provides the power necessary to heat the plasma to the operating temperature. Using a DT fuel cycle, the power $P_{n}$ associated with the neutrons resulting from the fusion reaction is converted to electric power at an efficiency of $\eta_{t}$, while the charged particle power $P_{c}$ that emerges from that end of the mirror which does not serve as a nozzle, is converted to electric power by a direct converter with efficiency $\eta_{D}$. Noting that

$$
P_{c}=\frac{1}{2}\left[(1+Q) \eta_{i} P_{i n j}-P_{n}\right]
$$

and realizing that there is a critical $Q$-value such that the gross electric power produced, $P_{G}$, is exactly equal to the injector power $P_{i n j}$, it is quite straight forward to show that it has the value

$$
Q=\frac{1-\frac{1}{2} \eta_{i} \eta_{D}}{\frac{1}{2} \eta_{i} \eta_{D}-\frac{\left(P_{n}+P_{r}\right)}{P_{f}}\left(\frac{1}{2} \eta_{D}-\eta_{t}\right)}
$$

with $P_{r}$ representing the total radiated power. Since $\eta_{i}$ is usually taken as unity, and for $\eta_{D}=0.9$ and $\eta_{t}=0.45, Q$ has the value 1.22 given in Table 1. It is clear that the value of $Q$ is quite sensitive to the efficiencies of the various components; for example it assumes the value of 1.664 when $\eta_{D}=$ 0.9 and the thermal efficiency is reduced to $30 \%$. As illustrated in Table 1 , the $Q$-value dictates the values of the various parameters that characterize the propulsion system including the size and the total mass.

\section{Application to Space Missions}

As a measure of the propulsive effectiveness of

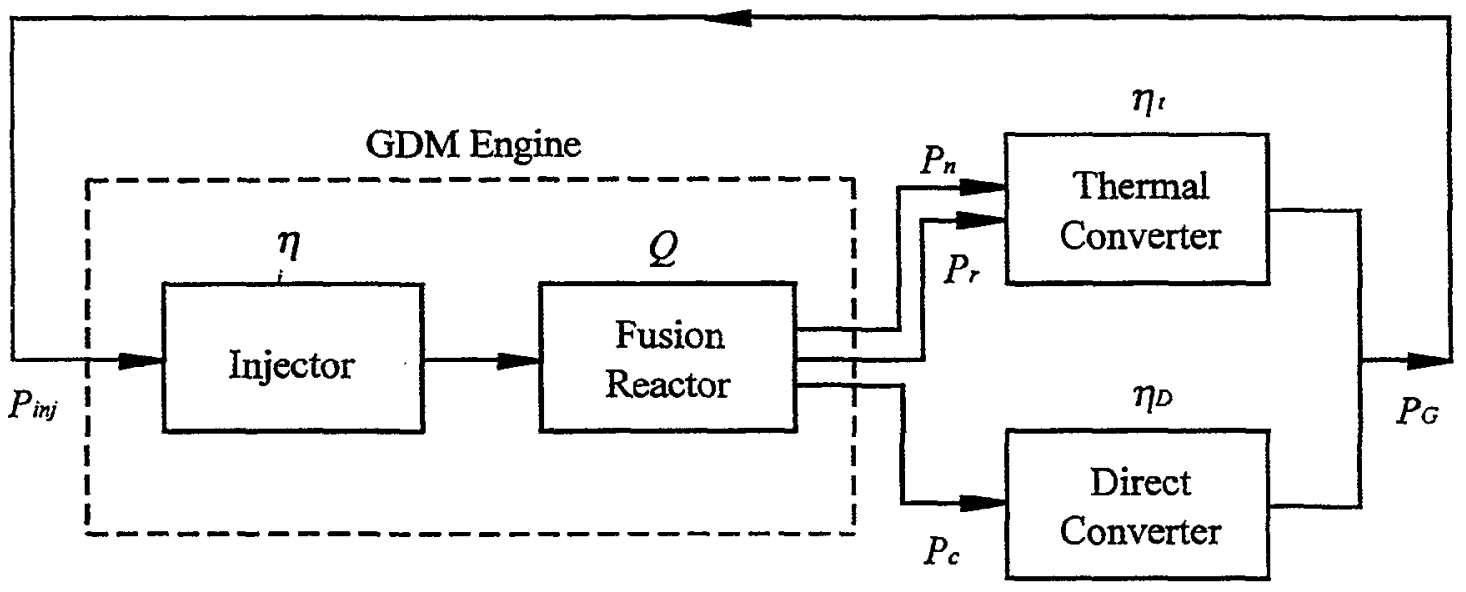

Fig. 2. Power flow diagram for GDM rocket 
Table 2

Solar space missions

\section{Parameters}

Gain factor $Q=1.22$

Plasma density $n=1 \times 10^{16} \mathrm{~cm}^{-3}$

Mirror ratio $R=100$

Plasma temperature $T=10 \mathrm{keV}$

Plasma beta (vacuum) $\beta=0.95$

Plasma length $L=44 \mathrm{~m}$

Plasma radius $r_{p}=5 \mathrm{~cm}$

Engine mass $m_{E}=101 \mathrm{Mg}$

\begin{tabular}{|c|l|c|c|c|c|c|c|}
\hline \multirow{2}{*}{$\delta$} & \multirow{2}{*}{ Type } & \multicolumn{2}{|c|}{ Mars } & \multicolumn{2}{c|}{ Jupiter } & \multicolumn{2}{c|}{ Pluto } \\
\cline { 3 - 8 } & $\begin{array}{c}\text { Propellant } \\
\text { mass (Mg) }\end{array}$ & $\begin{array}{c}\text { Trip time } \\
\text { (day) }\end{array}$ & $\begin{array}{c}\text { Propellant } \\
\text { mass (Mg) }\end{array}$ & $\begin{array}{c}\text { Trip time } \\
\text { (day) }\end{array}$ & $\begin{array}{c}\text { Propellant } \\
\text { mass (Mg) }\end{array}$ & $\begin{array}{c}\text { Trip time } \\
\text { (day) }\end{array}$ \\
\hline \multirow{3}{*}{0} & Fly-by & 10.3 & 59 & 29.8 & 170 & 90.5 & 517 \\
& Rendezvous & 14.7 & 84 & 42.2 & 241 & 128.3 & 733 \\
& Round trip & 29.6 & 169 & 86.4 & 494 & 273.9 & 1566 \\
\hline \multirow{3}{*}{0.25} & Fly-by & 10.7 & 61 & 30.6 & 175 & 93.0 & 532 \\
& Rendezvous & 15.1 & 86 & 43.4 & 248 & 131.9 & 754 \\
& Round trip & 30.4 & 174 & 88.8 & 508 & 281.1 & 1607 \\
\hline \multirow{3}{*}{0.5} & Fly-by & 11.0 & 63 & 31.5 & 180 & 95.5 & 546 \\
& Rendezvous & 15.5 & 89 & 44.6 & 255 & 135.4 & 774 \\
& Round trip & 31.3 & 179 & 91.2 & 522 & 288.0 & 1647 \\
\hline
\end{tabular}

GDM we apply the results obtained above to three types of missions to the planets Mars, Jupiter and Pluto with the latter selected because of its position as the outermost planet in the solar system. In making this assessment we employ simple trajectories based on a constant thrust, continuous burn acceleration/deceleration profiles for which we can use the standard rocket equation to compute the travel time. Under these assumptions the round trip journey time can be written as [7]

$$
\tau_{R T}=\frac{4}{g I_{s p}}+4 \sqrt{\frac{D m_{f}}{F}}
$$

where $D$ is the one-way linear distance between the points of travel, $g$ the Earth's gravitational acceleration, $F$ the thrust, $I_{s p}$ the specific impulse, and $m_{f}$ the final (dry) mass of the vehicle. For a one-way rendezvous mission the corresponding expression is

$$
\tau_{O R}=\frac{g I_{\text {sp }}}{F}\left(m_{i}-m_{f}\right)
$$

with the initial mass, $m_{i}$, given by

$$
m_{i}=\left[\sqrt{m_{f}}+\frac{\sqrt{D F}}{g I_{s p}}\right]^{2} .
$$

In addition to these two missions we include a flyby mission, and in all cases we calculate the propellant mass and travel time for three values of $\delta$ which represents the payload mass as fraction of the engine mass. The results are displayed in Table 2.

\section{Conclusion}

We have examined in this paper a propulsion device based on the gasdynamic mirror (GDM) fusion system and shown, on the basis of physics and engineering considerations, that it is realizable with almost present-day or near-term technology. It is capable of producing propulsive parameters that could make human exploration of interplanetary and interstellar space achievable sometime in the near future. Its geometric simplicity, ease of assembly on Earth or in space, and its capability of being self-sustaining, once it is ignited, makes it an extremely attractive propulsion system that can meet the stringent 
requirements of space travel. As can be seen from Table 1, the power required to heat the plasma is quite large and can perhaps be supplied only by a fission reactor which, if included, will make the vehicle very massive and complicated. It may allow GDM to operate at $Q<1$ [8] but the additional masses of the required components will truly exacerbate the total mass. Instead, we have demonstrated in this paper that a self-sustaining GDM can be quite modest in size and yet effective as a propulsion system. When applied to several missions of interest in the solar system, we have seen that with a self-sustaining GDM they can be carried out in relatively short times; and in the case of the outermost planet, Pluto, we have shown that round trip manned missions can be done in about four years.

\section{References}

[1] R. H. Hyde, "A Laser Fusion Rocket for Interplanetary Propulsion," AIF paper 83-396, 34th International Astronautical Conference, Budapest, October 1983

[2] T. Kammash and D. L. Galbraith, J. Propulsion and Power 6, 412 (1990)

[3] T. Kammash and M.-J. Lee, J. Propulsion and Power 11, 544 (1995)

[4] R. F. Post, Nuclear Fusion 27, 1579 (1987)

[5] V. P. Nagornyi, D. D. Ryutov and G. V. Stupakov, Nuclear Fusion 24, 1421 (1984)

[6] D. P. Kilcrease and R. C. Kirkpatrick, Nuclear Fusion 28, 1465 (1988)

[7] T. Kammash, Acta Astronautica 34, 17,(1994)

[8] T. Kammash and M.-J. Lee, "Fission-Assisted Gasdynamic Fusion Propulsion System," submitted to Fusion Technology 